\title{
THEMA
}

théorie économique,

modélisation et applications

THEMA Working Paper n²020-01 CY Cergy Paris Université, France

\section{Dummy players and the quota in weighted voting games: Some further results}

Fabrice Barthelemy, Mathieu Martin 


\title{
Dummy players and the quota in weighted voting games: some further results*
}

\author{
Fabrice BARTHELEMY ${ }^{\dagger}$ and Mathieu MARTIN ${ }^{\ddagger}$
}

\begin{abstract}
This paper is a companion paper of Barthélémy et al. (2019) which studies the role of the quota on the occurrence of "dummy" players in small weighted voting games (i.e., in voting games with 3,4 or 5 players). We here extend the results obtained in this paper by considering voting games with a larger number of players (up to 15). It is shown that the probability of having a player without voting power is very sensitive to the choice of the quota and the quota values that minimize this probability are derived.
\end{abstract}

\section{JEL classification: C7, D7}

Keywords: Cooperative game theory, weighted voting games, dummy player, probability of voting paradoxes.

${ }^{*}$ Financial support from the ANR SOLITER is gratefully acknowledged. This research has been also developed within the center of excellence MME-DII (ANR-11-LBX-0023-01). Warm thanks are due to Dominique Lepelley for his valuable help.

${ }^{\dagger}$ CEMOTEV, Université de Versailles Saint-Quentin en Yvelines, Economics, FRANCE. E-mail: fabrice.barthelemy@uvsq.fr

${ }^{\ddagger}$ THEMA, Université de Cergy-Pontoise, FRANCE. E-mail: mathieu.martin@u-cergy.fr 


\section{Introduction}

In this paper, we compute the probability of having a dummy player in a weighted voting game. A dummy player is a player who has no power or no influence in a collective decision. The most famous example is probably the well-known case of Luxembourg in the Council of Ministers of the EU between 1958 and 1973. It proves that it is possible to have a weight different from 0 (Luxembourg had one vote) and absolutely no power in the decisions. Of course, such decisions are undesirable but we should not worry about them if it could be shown that their occurrence is rare. In the classical case of majority games, Barthélémy et al. (2013) show that the probability of having a dummy player is unfortunately far from 0 . To illustrate, this probability can reach about $50 \%$ for 4,5 or 6 players; for more than 6 players, the probability decreases but we have to consider more than 15 players for obtaining results lower than $1 \%$. Do these negative results hold when other quotas are considered?

Does this negative result hold when other quotas are considered? Our goal is to determine the quota values which minimize this probability and then to answer to the previous question.

We know that under unanimity rule, each player has a veto power and hence is not a dummy. Can we find other quotas for which the risk of having a dummy player is zero? We give a negative answer to this question. Consequently, the major part of this study is devoted to the determination of quota values that minimize (or maximize) the probability of having a dummy player. To the best of our knowledge, this issue has never been tackled in the literature, with the exception of Barthélémy [et al. (2019), a companion paper. The results we obtain in the second part of the current paper their results, where some analytical results are given for 3, 4 and 5 players. Some general formulas are proposed typically in the spirit of a huge literature on this subject (see, for example Gehrlein and Lepelley 2017). Even if this approach is the most interesting, it is limited because the calculus are too complicated when the number of players increases. Moreover, for 4 and 5 players, contrary to the case with only 3 players, only limiting representations are obtained, when the sum of the weights (the number of seats for example) tends to infinity. It is why we proceed here by computer enumeration or simulations, in function of the size of the variables.

The paper is organized as follows: in the second section, our notation, definitions and assumptions are introduced. In the third section, we present general preliminary results which show that, from a theoretical point of view, avoiding a dummy player seems to be complicated. Of course, it does not mean that the probability of such an event is high, it only means that it is very easy to find a game such that there is a dummy player. Some numerical results for the probability of having at least one dummy player are then obtained in section 4. The main conclusions of our study are summarized and discussed in the fifth 
section.

\section{Notation, definitions and assumptions}

The formal framework of this study is the same as in Barthélémy et al. (2019). The reader is referred to this companion paper for further (but here unnecessary) details on this framework.

A voting game is a pair $(N, W)$ where $N=\{1,2, \ldots, n\}$ is the set of $n$ players (or voters) and $W$ the set of winning coalitions, that is the set of groups of players which can enforce their decision. We consider the class of weighted voting games $\left[q ; w_{1}, w_{2}, \ldots, w_{n}\right]$, where $q$ is the quota needed to form a winning coalition and $w_{i}$ is the number of votes (weight) of the $i$ th player; we assume that $q$ and $w_{i}$ are integers. A coalition $S$ is winning if and only if $\sum_{i \in S} w_{i} \geq q$. The total number of votes, $\sum_{i \in N} w_{i}$, is denoted by $w$. A particular case is the majority game where $q_{m a j}=\frac{w}{2}+1$ if $w$ is even and $q_{m a j}=\frac{w+1}{2}$ if $w$ is odd. We assume that the game is proper, that is $q \geq q_{m a j}$. When $q=w$, we get the unanimity rule: each player has a veto power and is not a dummy. Our study will focus on the weighted voting games such that $q \leq w-1$. The relative quota, denoted $Q$ with $Q=q / w$, is used in the tables. We assume, without loss of generality, that $w_{1} \geq w_{2} \geq \ldots \geq w_{n} \geq 0$.

A player $i$ is a dummy player in a voting game $(N, W)$ if $S \in W$ implies $S \backslash\{i\} \in W$ for every $S \in W$. In words, player $i$ is never decisive in every winning coalition: the coalition wins with or without him (her). To illustrate, player 3 is a dummy in the weighted voting game $[5 ; 3,2,1]$. In voting power theory (see Straffin, 1994, and Felsenthal and Machover, 1998 , for a presentation), it means that this player has no power.

A weighted voting game is said to be admissible if each player has at least one vote $\left(w_{n} \geq 1\right)$ and never more than $q-1$ votes (there is no dictator). Of course, if player $j$ is a dummy player, then player $k$ with $k>j$ is also a dummy. Also notice that, as $w_{1} \leq q-1$ in an admissible weighted voting game, player 2 is never a dummy and, consequently, the maximum number of dummy players is equal to $n-2 .^{1}$

The purpose of this paper is to compute the probability of obtaining a dummy player, given $n, w$ and $q$ (or $Q$ ), and to derive the quota which minimizes this probability (denoted by $\underline{Q}$ ) and the quota which maximizes this probability (denoted by $\bar{Q}$ ). The probability of having at least one dummy player is denoted by $P(w, n, q)$ or $P(w, n, Q)$ when $w$ is finite and $P(n, Q)$ when $w$ is infinite.

In order to compute $P(w, n, q)$ (or $P(w, n, Q)$ or $P(n, Q)$ ), we consider a particular probabilistic model called IAC (Impartial Anonymous Culture) which is one of the most

1. In the particular case where $q=q_{m a j}$, not only player 2 but also player 3 cannot be a dummy; see Proposition 1 in Barthélémy et al. (2013). In this case, the maximum number of dummy players is $n-3$. 
often used in such problems where the likelihood of a voting event is to be calculated (see, for instance, Lepelley et al., Diss et al., 2012, or Courtin et al., 2014).

In the current context, using this model is tantamount to assume that, $n, w$ and $q$ being given, all the admissible distributions of the $w_{i}$ 's, i.e. all the distributions such that $\left(q-1 \geq w_{1} \geq w_{2} \geq \ldots \geq w_{n} \geq 1\right.$ and $\left.\sum_{i \in N} w_{i}=w\right)$ are equally likely to occur.

\section{Preliminary results}

We present preliminary results concerning the general possibility of obtaining a dummy player in a weighted voting game. We know that there is no dummy player when $q=w$. We also know from Leech (2002) (see also Barthélémy et al., 2013) that, in the three-player case, there is no dummy player when $q=q_{m a j}$. Can we find some other values of $q$ and $n$ (the number of players) for which the "dummy paradox" never occurs? The following propositions give a negative answer to this question (as soon as $w$, the total number of votes, is not very small).

Proposition 1 For all $n, q$ and $w$ such that $n \geq 4, q_{m a j} \leq q \leq w-1$ and $w \geq 6 n-12$, there exists a game $\left[q ; w_{1}, w_{2}, \ldots, w_{n}\right]$ where player $n$ is a dummy player.

This result is a consequence of the following lemmas :

Lemma 1 For all $n$ and $w$ such that $n \geq 4, w \geq 6 n-12$ and $q=\frac{w}{2}+1$, there exists a game $\left[\frac{w}{2}+1 ; w_{1}, w_{2}, \ldots, w_{n}\right]$ where player $n$ is a dummy.

Proof. Consider the following game : $\left[\frac{w}{2}+1 ; \frac{w}{2}-2, \frac{w-6 n+26+a}{4}, \frac{w-6 n+26-a}{4}, 3,3, \ldots, 3,1\right]$ with $a=0$ if $n$ is odd (even) and $\frac{w}{2}$ is even (odd) and $a=2$ if $n$ is odd (even) and $\frac{w}{2}$ is odd (even). Furthermore, the number of individuals whose weight is 3 is equal to $n-4$.

We have to show that (1) $q>w_{1} \geq w_{2} \geq \ldots \geq w_{n}$ and, since $w_{n}=1$, we have to show that (2) there is no coalition $S \subseteq N \backslash\{n\}$ such that $\sum_{i \in S} w_{i}=q-1$. Indeed, this is the only situation for which player $n$, with one vote, is not a dummy player.

(1) Clearly $q>w_{1}$ and $w_{2} \geq w_{3}$, thus we have just to show that $w_{1} \geq w_{2}$ and $w_{3} \geq 3$ (if $n=4$ we have to show that $w_{3} \geq 1$ which is obvous). Assume that $w_{2}>w_{1}$ then we obtain $w<34-6 n+a$. By hypothesis, we have $w \geq 6 n-12$ thus $w_{2}>w_{1}$ if $34-6 n+a>6 n-12$ or $n<\frac{46+a}{12} \leq 4$, a contradiction of $n \geq 4$. QED

Assume now that $w_{3}<3$, it means that $\frac{w-6 n+26-a}{4}<3$ or $w<6 n-14+a$. By hypothesis, $w \geq 6 n-12$ thus $6 n-14+a>w \geq 6 n-12$ and then $a>2$ which is impossible. Thus $w_{3} \geq 3$.

(2) Consider a coalition $S \subseteq N \backslash\{n\}$ with $1 \in S$. Since $w_{3} \geq 3$ we have $\sum_{i \in S} w_{i} \geq q$ except if $S=\{1\}$. Since $w_{1}=q-3$, it is never possible to obtain a total weight equal to 
$q-1$ with player 1 belonging to $S$. Assume now that player 1 does not belong to $S$ : we have $\sum_{i=2}^{n-1} w_{i}=q$. Since $w_{i} \geq 3, i=2,3, \ldots, n-1$, it is not possible to obtain a total weight equal to $q-1$ in $S$. QED

Lemma 2 For all $n, q$ and $w$ such that $n \geq 4, w>4 n-8, \frac{w}{2}+1<q \leq w-2 n+5$, there exists a game $\left[q ; w_{1}, w_{2}, \ldots, w_{n}\right]$ where player $n$ is a dummy.

Proof. Consider the following game : $[q ; q-2, w-q-2 n+7,2,2, \ldots, 2,1]$. We have to show that (1) $q>w_{1} \geq w_{2} \geq \ldots \geq w_{n}$ and, since $w_{n}=1$, we have to show that (2) there is no coalition $S \subseteq N \backslash\{n\}$ such that $\sum_{i \in S} w_{i}=q-1$.

(1) We have just to show that $w_{1} \geq w_{2}$ and $w_{2} \geq 2$. Assume that $w_{2}>w_{1}$. Thus $w-q-2 n+7>q-2$ or $n<\frac{w-2 q+9}{2}$. Since $n \geq 4$, we have $4 \leq n<\frac{w-2 q+9}{2}$ and then $q \leq \frac{w+1}{2}$, a contradiction.

Furthermore, $w_{2} \geq 2$ if $w-q-2 n+7 \geq 2$ or $q \leq w-2 n+5$, which is true by hypothesis.

(2) Consider a coalition $S \subseteq N \backslash\{n\}$ with $1 \in S$. Since $w_{2} \geq 2$ we have $\sum_{i \in S} w_{i} \geq q$ except if $S=\{1\}$. Since $w_{1}=q-2$, it is never possible to obtain a total weight equal to $q-1$ with player 1 belonging to $S$. Assume now that $1 \notin S$, thus we have $\sum_{i=2}^{n-1} w_{i}=w-q+1$. But $w-q+1=q-1$ implies $q=\frac{w}{2}+1$, a contradiction. Therefore there is no $\bar{S} \subset S$ such that $\sum_{i \in \bar{S}} w_{i}=q-1$.

Remark that the condition $w>4 n-8$ guarantees that $w-2 n+5>\frac{w}{2}+1$.

Lemma 3 For all $n, q$ and $w$ such that $n \geq 4, w \geq 5 n-12$ and $w-2 n+6 \leq q \leq w-n+2$, there exists a game $\left[q ; w_{1}, w_{2}, \ldots, w_{n}\right]$ where player $n$ is a dummy.

Proof. Consider the following game : $[q ; q-n+1, w-q+1,1, \ldots, 1]$. We have to show that (1) $q>w_{1} \geq w_{2} \geq \ldots \geq w_{n}$ and, since $w_{n}=1$, we have to show that (2) there is no coalition $S \subseteq N \backslash\{n\}$ such that $\sum_{i \in S} w_{i}=q-1$.

(1) We have just to show that $w_{1} \geq w_{2}$ and $w_{2} \geq 1$. Assume that $w_{2}>w_{1}$, thus $q-n+1<w-q+1$ or $q<\frac{w+n}{2}$. We know that $q \geq w-2 n+6$ thus $\frac{w+n}{2}>q \geq w-2 n+6$, that is to say $w<5 n-12$, a contradiction. It is obvious that $w_{2} \geq 1$.

(2) Remark that $w_{1}+w_{3}+w_{4}+\ldots+w_{n-1}=q-2$. Thus players 1 and 2 must belong to the coalition $S \subseteq N \backslash\{n\}$ if we want $\sum_{i \in S} w_{i} \geq q-1$. We have $w_{1}+w_{2}=w-n+2$ and by hypothesis, $q \leq w-n+2$, thus $w_{1}+w_{2} \geq q$. It is not possible to obtain a total weight equal to $q-1$. QED

Lemma 4 For all $n, q$ and $w$ such that $n \geq 4, w \geq 4 n-9$ and $w-n+3 \leq q \leq w-1$, there exists a game $\left[q ; w_{1}, w_{2}, \ldots, w_{n}\right]$ where player $n$ is a dummy.

Proof. Consider the game $[q ; q-(n-w+q-1)(w-q+1), w-q+1, \ldots, w-q+1,1 \ldots, 1]$, knowing that the number of players with a weight equal to $w-q+1$ is equal to $n-w+q-1$ 
and the number of players with a weight equal to 1 is equal to $w-q$. We have to show that (1) $q>w_{1} \geq w_{2} \geq \ldots \geq w_{n}$ and, since $w_{n}=1$, we have to show that (2) there is no coalition $S \subseteq N \backslash\{n\}$ such that $\sum_{i \in S} w_{i}=q-1$.

(1) Assume $w_{1} \geq q$. Thus we have $q-(n-w+q-1)(w-q+1) \geq q$ or $(n-w+q-$ 1) $(w-q+1) \leq 0$ which is impossible since $(n-w+q-1)>0$ and $(w-q+1)>0$.

Let us show now that $w_{1} \geq w_{2} . w_{2}$ is maximized when $q$ is minimized, that is to say when $q=w-n+3$. Thus $\bar{w}_{2}=n-2\left(\bar{w}_{2}\right.$ is the highest value of $\left.w_{2}\right)$. We then obtain the lowest value of $w_{1}$, denoted $\underline{\mathrm{w}}_{1}$ such that $\underline{\mathrm{w}}_{1}=w-3 n+7$ ( $q$ is replaced by $w-n+3$ in $\left.w_{1}\right)$. We have always $w_{1} \geq w_{2}$ if $\underline{\mathrm{w}}_{1} \geq \bar{w}_{2}$ that is to say $w-3 n+7 \geq n-2$ or $w \geq 4 n-9$ which is true by hypothesis.

(2)Assume that there exists a coalition $S$ such that $\sum_{i \in S} w_{i}=q-1$ knowing that player $n$ does not belong to $S$. Let $S_{1}=\{1,2, \ldots, n-w+q\}$ be a coalition in which any player $i$ is such that $w_{i} \neq 1$. We have $\sum_{i \in S_{1}} w_{i}=q \neq q-1$ thus $S_{1} \neq S$. It means that player 1 or player 2 (w.l.o.g) does not belong to $S$. Let $S_{2}=\{1,3, \ldots, n-1\}$ be the coalition with all the players except 2 and $n$. We have $\sum_{i \in S_{2}} w_{i}=w-(w-q+1)-1$ or $\sum_{i \in S_{2}} w_{i}=q-2 \neq q-1$. Thus $S_{2} \neq S$ which implies that 2 belongs to $S$. Therefore any $i$ such that $w_{i}=w-q+1$ belongs to $S$ and since $w_{1} \geq w_{2}, 1$ belongs to $S$ as well. It means that $\sum_{i \in S} w_{i} \geq q$, a contradiction.

Proof of proposition 1. The different constraints given by the lemmas 1 to 4 are the following : $w \geq 6 n-12$ and $q=\frac{w}{2}+1$ (lemma 1), $w>4 n-8$ and $\frac{w}{2}+1<q \leq w-2 n+5$ (lemma 2), $w \geq 5 n-12$ and $w-2 n+6 \leq q \leq w-n+2$ (lemma 3 ) and $w \geq 4 n-9$ and $w-n+3 \leq q \leq w-1$ (lemma 4 ). It is easy to verify that all possible quotas for admissible games (from $\frac{w}{2}+1$ to $w-1$ ) are considered. Furthermore, a very simple calculus shows that the more restrictive constraint is $w \geq 6 n-12$. QED

Proposition 1 only deals with at least 4 players. Proposition 2 presents a similar result for the 3-player case for $q>q_{m a j}$.

Proposition 2 In the three-player case, there exists a game $\left[q ; w_{1}, w_{2}, w_{3}\right]$ such that player 3 is a dummy for each value of $q, q \leq w-1$ and $q \neq q_{m a j}$.

Proof. Consider the game $[q ; q-2, w-q+1,1]$. We have to show that (1) $w_{1} \geq w_{2} \geq w_{3}$ and (2) player 3 is a dummy player i.e. $w_{1}+w_{2} \neq q-1$.

(1) $w_{2} \geq w_{3}$ if $w-q+1 \geq 1$ or $w \geq q$ which is always true. Furthermore, $w_{1} \geq w_{2}$ if $q-2 \geq w-q+1$ or $q \geq \frac{w+3}{2}$ which is always true since $q \neq q_{m a j}$.

(2) We have $w_{1}+w_{2}=w-1$ which is different from $q-1$ since $q<w$. QED 


\section{Numerical results}

From Barthélémy et al. (2019), we know that, in weighed voting games with 3, 4 or 5 players, the probability of having a dummy player is very sensitive to the choice of the quota and can be very high; in addition, it turns out that, except for quotas very close to 1, increasing the quota does not decrease the probability of dummy players. To what extent can we generalize these conclusions to voting games with a larger number of players? It is the question we investigate in this section.

Our results, presented in tables and graphs, are obtained with one of the two following approaches. The first one gives exact results whereas the second one is based on simulations and provides estimated probabilities. Note that for small numbers of players, these results corroborate what we have obtained analytically in Barthélémy et al. (2019).

Exact computations are done by considering the exhaustive list of all possible vectors of weights for a given number $w$ of votes. For all these vectors $\left(w_{1}, \ldots, w_{n}\right)$, we check whether or not the last player is decisive (remember that $w_{1} \geq w_{2} \geq \ldots \geq w_{n}$ ). To do this, we use the classical Banzhaf power index ${ }^{2}$ since a player, by construction, is a dummy if his (her) index is equal to zero. We compute this index using a generating functions approach which leads to exact values (this point is fundamental because we are looking for an index with a zero value, which prohibits the use of approximation methods). Finally, the exact probability of having at least one dummy player is the ratio between the number of times the last player is never decisive and the number of vectors $\left(w_{1}, \ldots, w_{n}\right)$ considered as admissible (in accordance with the assumed uniform distribution of weight vectors). Unfortunately, enumerating all these distributions is highly time consuming when the number of players and $w$ become large (see, for example, Barthélémy et al., 2011). It is the reason why we also resort to simulations.

Our simulations are based on random vectors of weights. The estimated probability of having at least one dummy player is then obtained by dividing the number of times the last player is never decisive by the number of vectors $\left(w_{1}, \ldots, w_{n}\right)$ randomly generated. In order to simulate the probability of a dummy player, two steps have to be considered. First, we have to simulate a vector of weights for a given $w$ and a given number of players $n$. This can be done by using for instance the Rancom algorithm proposed by Nijenhuis and Wilf (1978). Second, we have to check whether there is at least one dummy player in the weighted game associated to these weights. This is done as mentioned above by using the Banzhaf power index. Then repeating these two steps $k$ times gives the estimated probability which corresponds to the proportion of weighted games leading to a dummy player.

We analyze first the results for a finite total number of votes $w$. In a second step, we

2. For a clear and simple presentation, see Straffin (1994). 
will extend our study to the case where this total number of votes tends to infinity.

\subsection{Finite case}

We compute both exact and simulated probabilities. Tables 1 to 3 give the probability of having at least one dummy player according to given values of the quota $Q$, for weighted games with 3,5 and 10 players. ${ }^{3}$

The probabilities $P(n, w, Q)$ are not monotonic with respect to parameters $Q, w$ and $n$. However, we observe that the probability tends to 0 when $n$ increases, which is a particular illustration of the so-called Penrose's law (or Penrose's limit theorem). Penrose (1946, 1952) argues (without rigorous proof) that, if the number of players is large while the quota is fixed at half of the total weight, then the ratio between the voting powers of any two players, measured by their Banzhaf index, tends to the ratio between their weights. Lindner and Machover (2003) have shown that, if the Penrose's law is not always true, "experience suggests that counter-examples are atypical" and they conjecture that the theorem holds under rather general conditions, for large classes of weighted voting games, other values of the quota and other measures of voting power. Using simulations, Chang et al. (2006) conclude that if the result holds only for a quota of $50 \%$ when the Banzhaf index is considered, the Penrose's law remains valid for all values of the quota when power is measured by the Shapley-Shubik index. ${ }^{4}$ Let us notice that a dummy player with the Banzhaf index is a dummy player with the Shapley-Shubik index as well (and reciprocally). As there are no weights equal to zero by construction in our study, each player tends to get a positive power when $n$ tends to infinity and the probability of having a dummy player tends to zero. Figure 1 illustrates this result for $w=60$ and $Q=2 / 3,0.75,0.90,0.95 .^{5}$

Tables 1 to 3 report as well the optimal probabilities $P(n, w, \underline{Q}), P(n, w, \bar{Q})$ (denoted $P_{\min }$ and $\left.P_{\max }\right)$ and the corresponding quotas $\underline{Q}$ and $\bar{Q}$. For a given $w$, we compute all the quotas $Q$ running from majority to unanimity. More precisely, we consider all the quotas from $Q=0.50$ (corresponding to either $q=w / 2+1$ or $q=(w+1) / 2)$, to $Q=(w-1) / w$ (the closest quota to unanimity, $q=w-1$ ).

For instance, for $w=10$, we calculate the probability of having at least one dummy player for $q=6,7,8$ and 9 , leading to relative quotas equal to $0.5,0.6,0.7,0.8$ and $0.9 .{ }^{6}$ In this case, we get unanimity as soon as $q$ is greater than 9 and this implies that for all $Q>0.9, P(10, n, Q)=0 \%$.

3. This is an arbitrary choice. Any number of players, reasonably large, can be studied.

4. See Straffin (1994) for a presentation of this power index.

5. Obviously, other values of $w$ or $Q$ lead to the same kind of curves.

6. Note that $Q=0.5$ and $Q=0.6$ correspond to the same quota $q=6$. 


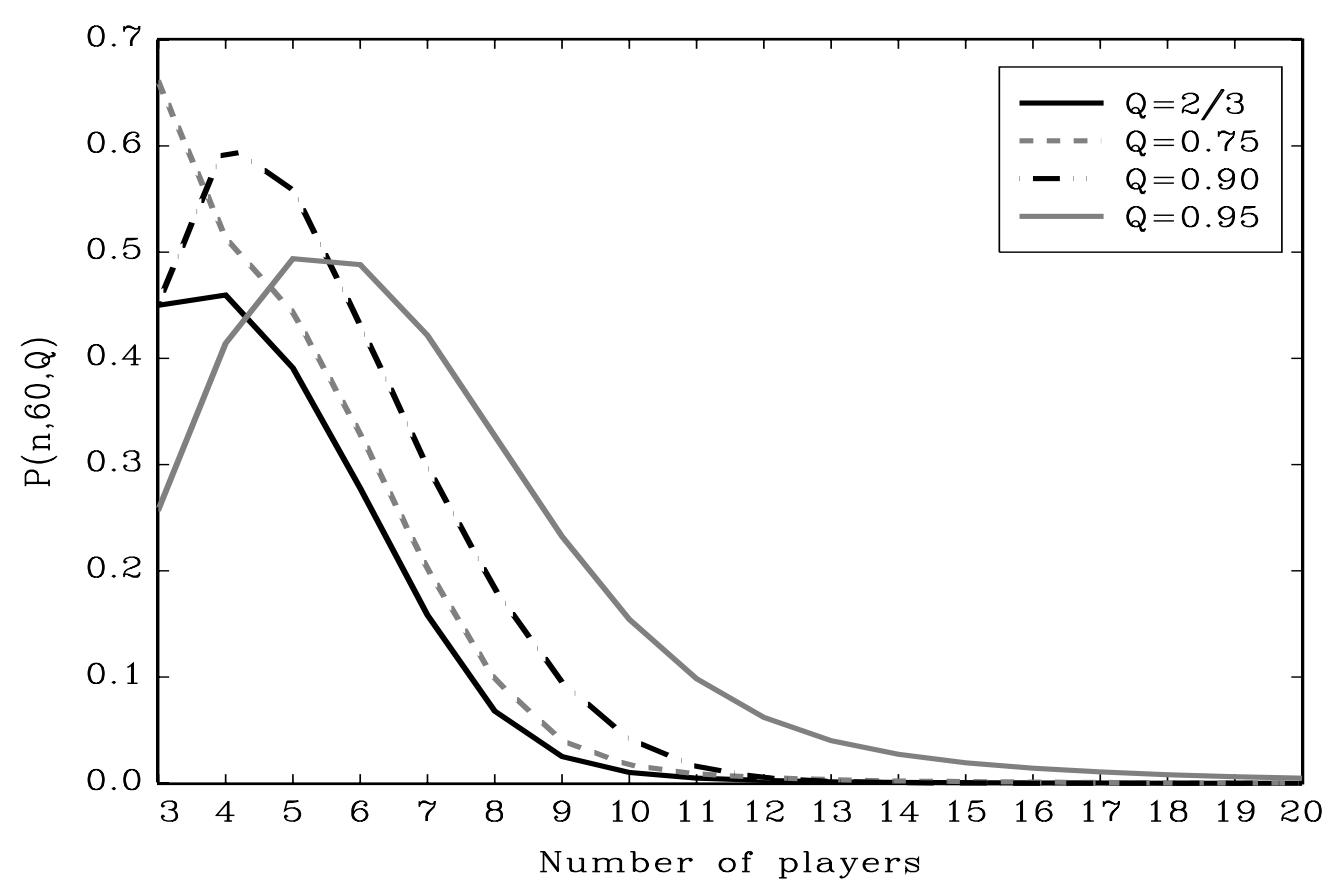

Figure 1 - Illustration of the Penrose's law for $w=60$

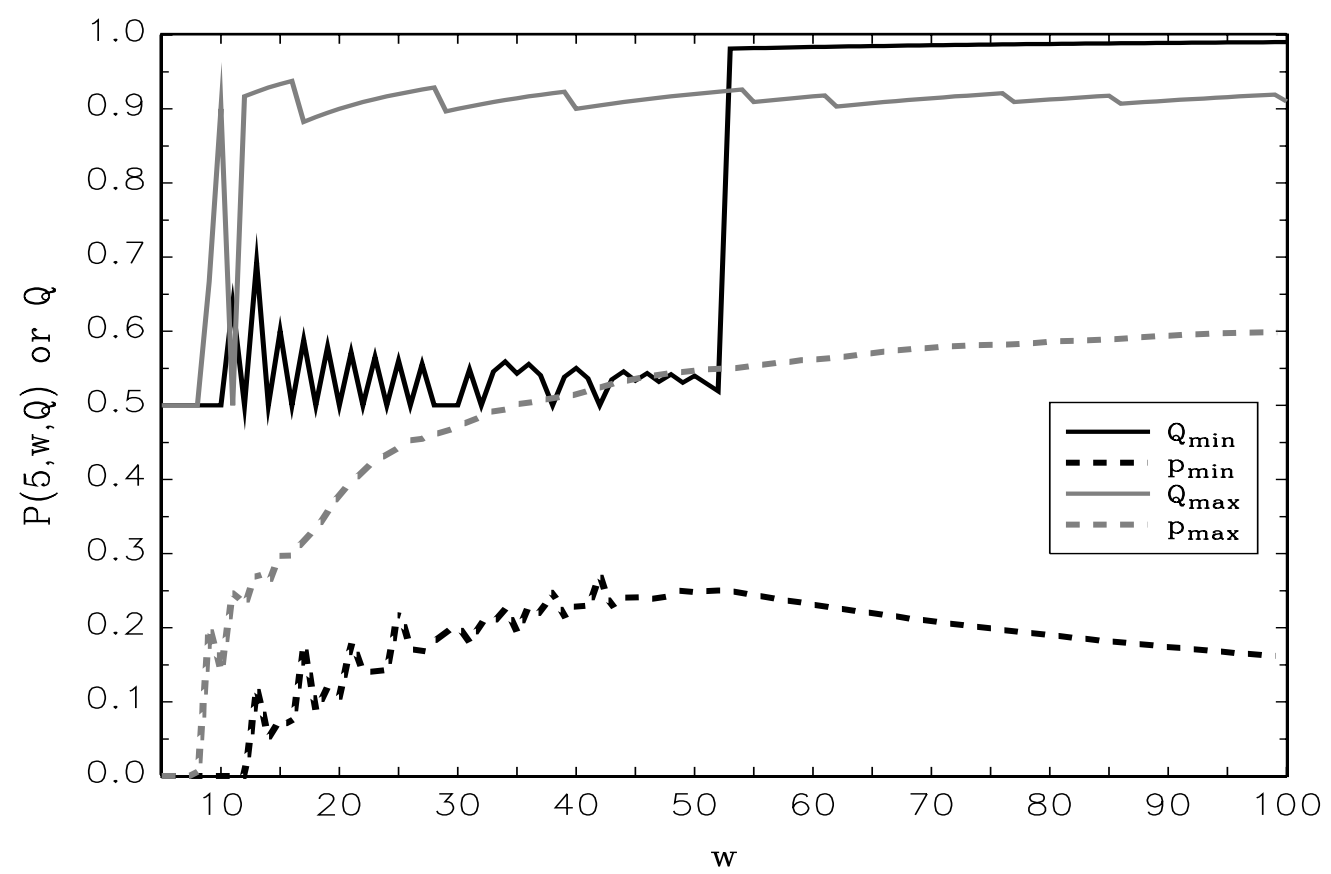

FIGURE 2 - 5-player case, smallest and highest probabilities of a dummy player according to $w$

Similarly, when considering $w=20$, the unanimity case is obtained as soon as $Q>0.95$ (corresponding to $q>19$ ). In Tables 1 to 3, the symbol '-' is represented for cases where 
the relative quota $Q$ is equivalent to the unanimity case $(q=w)$. Remark that we do not take into account the unanimity case in the computation of $P_{\min }$ (as mentioned above, unanimity leads to a zero probability).

As the number of different quotas may be high (49 possible quotas for $w=100$ ), only selected values of $Q$ are reported in Tables 1 to 3, with $Q \in\{0.5,2 / 3,0.75,0.8,5 / 6,0.9,0.95$, $0.98,0.99\}$. But $P_{\min }$ and $P_{\max }$ are computed using all the possible quotas. For instance, with $w=15$ and $n=5, P_{\min }=7.69 \%$ with a quota $Q=0.60$ (not reported in Table 2 ). If more than one quota $q$ lead to the smallest probability $P_{\min }, \underline{q}$ is the smallest value, and $\underline{Q}$ is the corresponding relative value. For the above example, each $Q \in[0.60,2 / 3[$ (corresponding to $\left[9 / 15,10 / 15\left[\right.\right.$ ) leads to the same probability $P_{\min }=7.69 \%$, and our convention gives $Q=0.60$.

Concerning $\underline{Q}$, a strange phenomenon is worth noticing. For small values of $w, \underline{Q}$ is close to the majority and when $w$ increases, $\underline{Q}$ suddenly tends to unanimity. Figure 2 illustrates this phenomenon in the 5-player case. The value $\tilde{w}$ of the total number of votes for which $Q$ becomes (almost) the unanimity instead of (almost) the majority increases with $n, n \geq 4$, the number of players. For instance $\tilde{w}=35$ in the 4-player case, $\tilde{w}=53$ in the 5 -player case, $\tilde{w}=87$ in the 6 -player case and $\tilde{w}>100$ when $n \geq 6$.

\subsection{Infinite case}

Limiting probabilities are simulated probabilities computed with high values of the total number of votes $w$.

Table 4 reports the estimated limiting probabilities from 3 to 15 players. Each row illustrates the Penrose's law : the probabilities tend to decrease when $n$ increases, as previously mentioned for the finite case. ${ }^{7}$ Figure 3 is given as an illustration of this remark with four values of $Q$.

Figure 4 presents the limiting probabilities plotted for 4, 6, 8, 10 and 15 players as a function of the quota $Q$. This Figure illustrates that fixing the quota at values higher than $50 \%$ (as $2 / 3$ or even $3 / 4$ ) does not lead to a smaller probability of having at least one dummy player. Moreover, in general, increasing the quota tends to increase this probability, except for values of $Q$ very close to 1 . This is particularly clear when the number of players is high.

7. Note however that for high values of $Q$, the convergence is not clear and more players are needed in order to recover Penrose's law. 


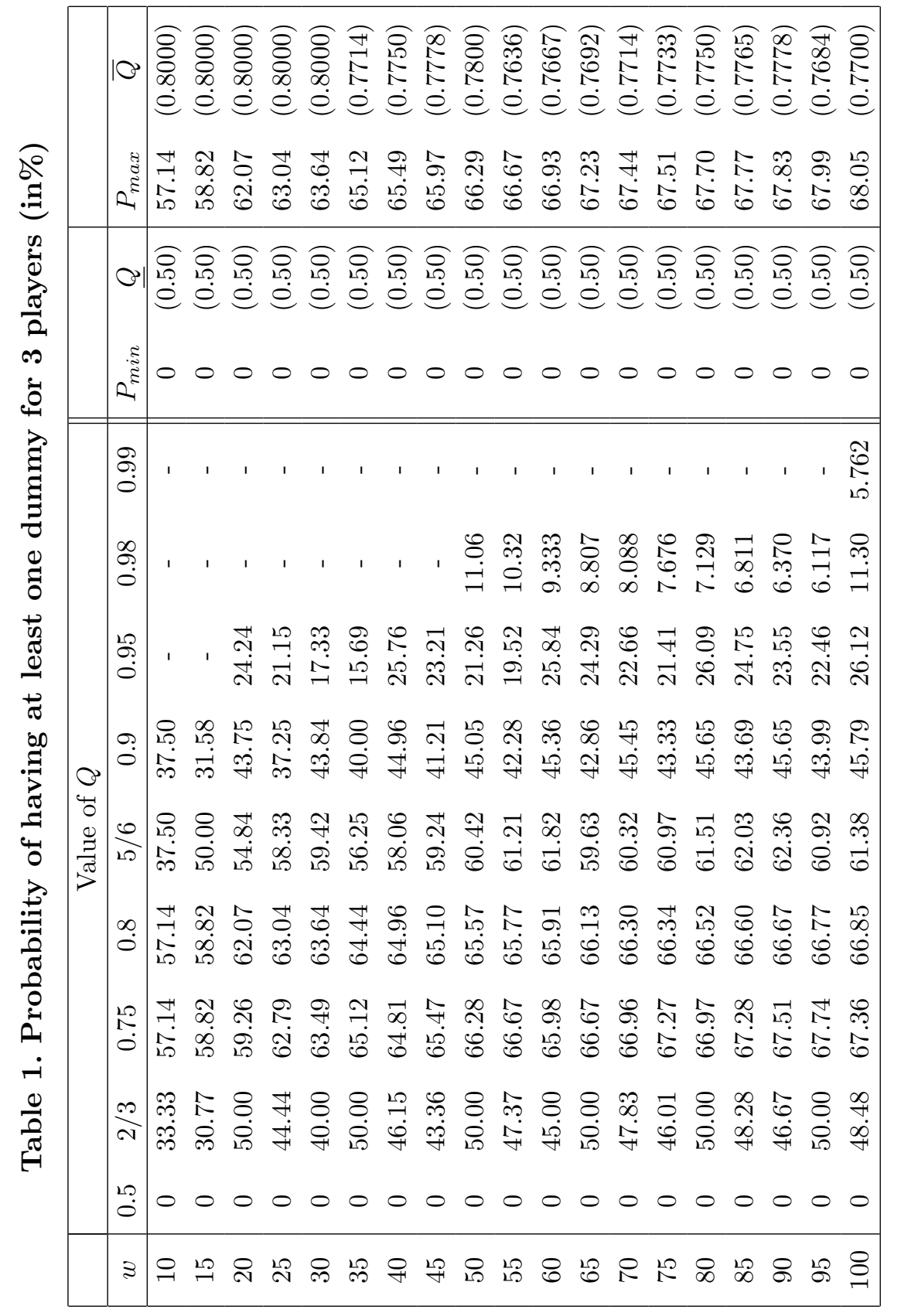




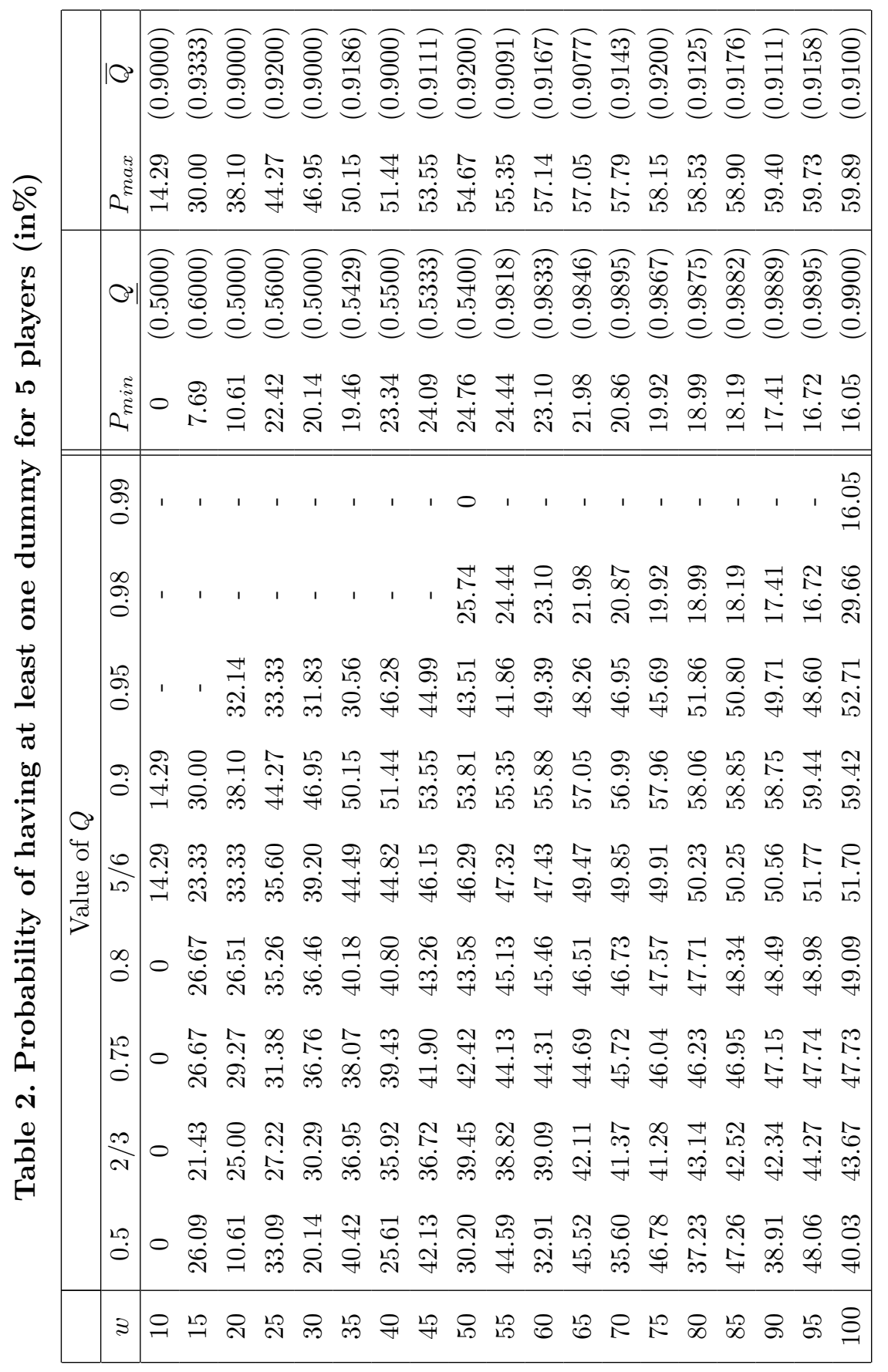




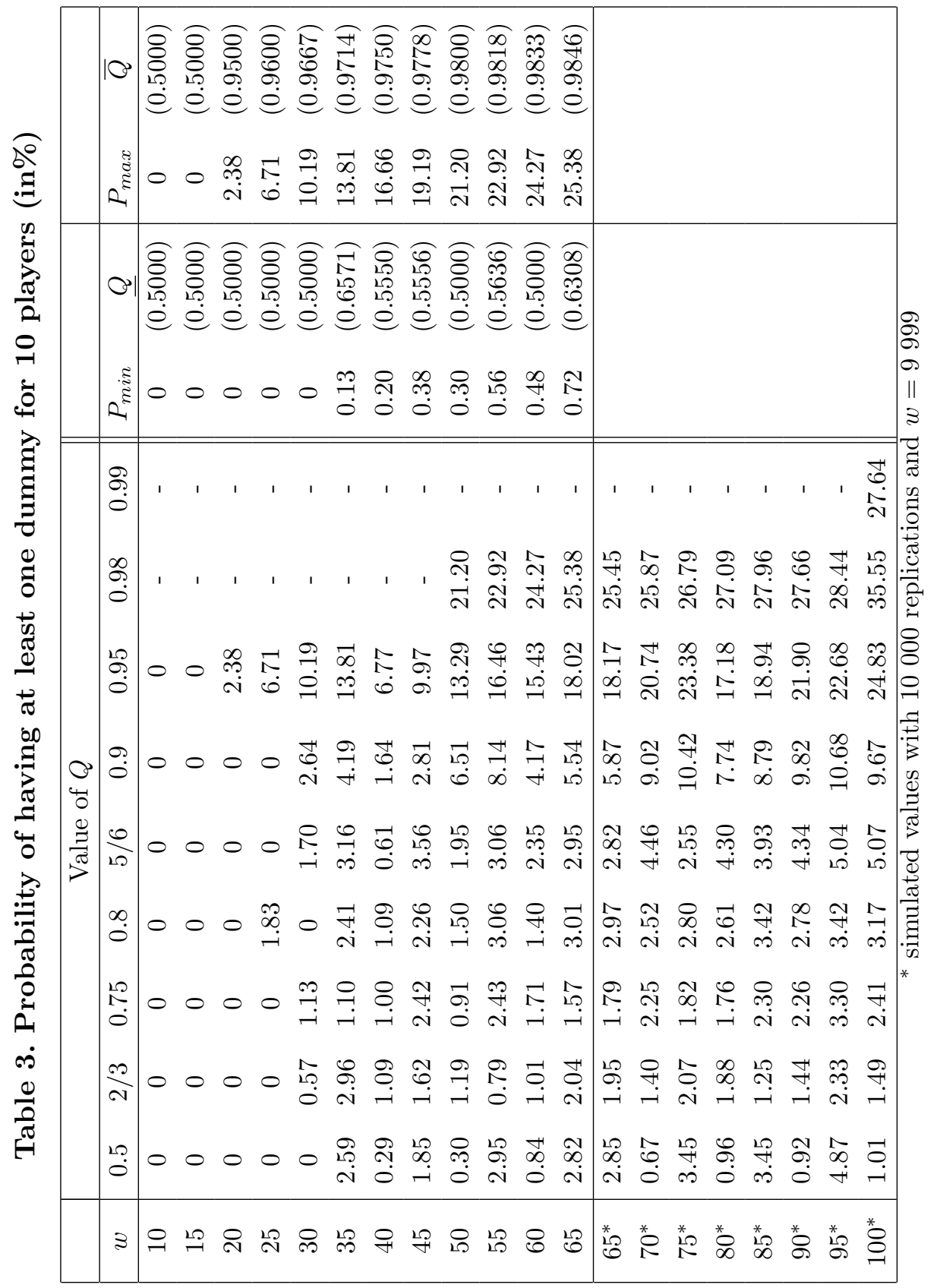




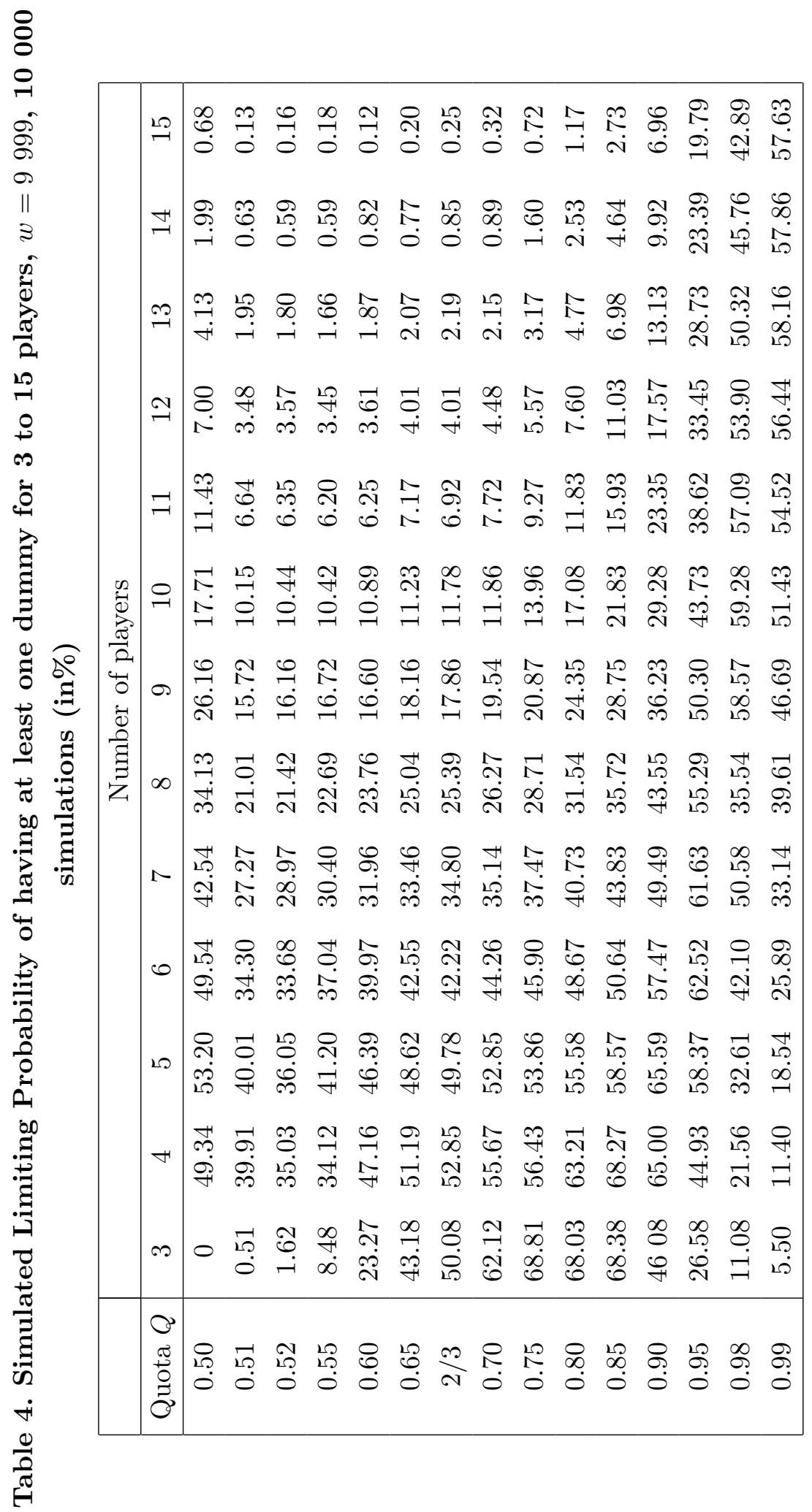




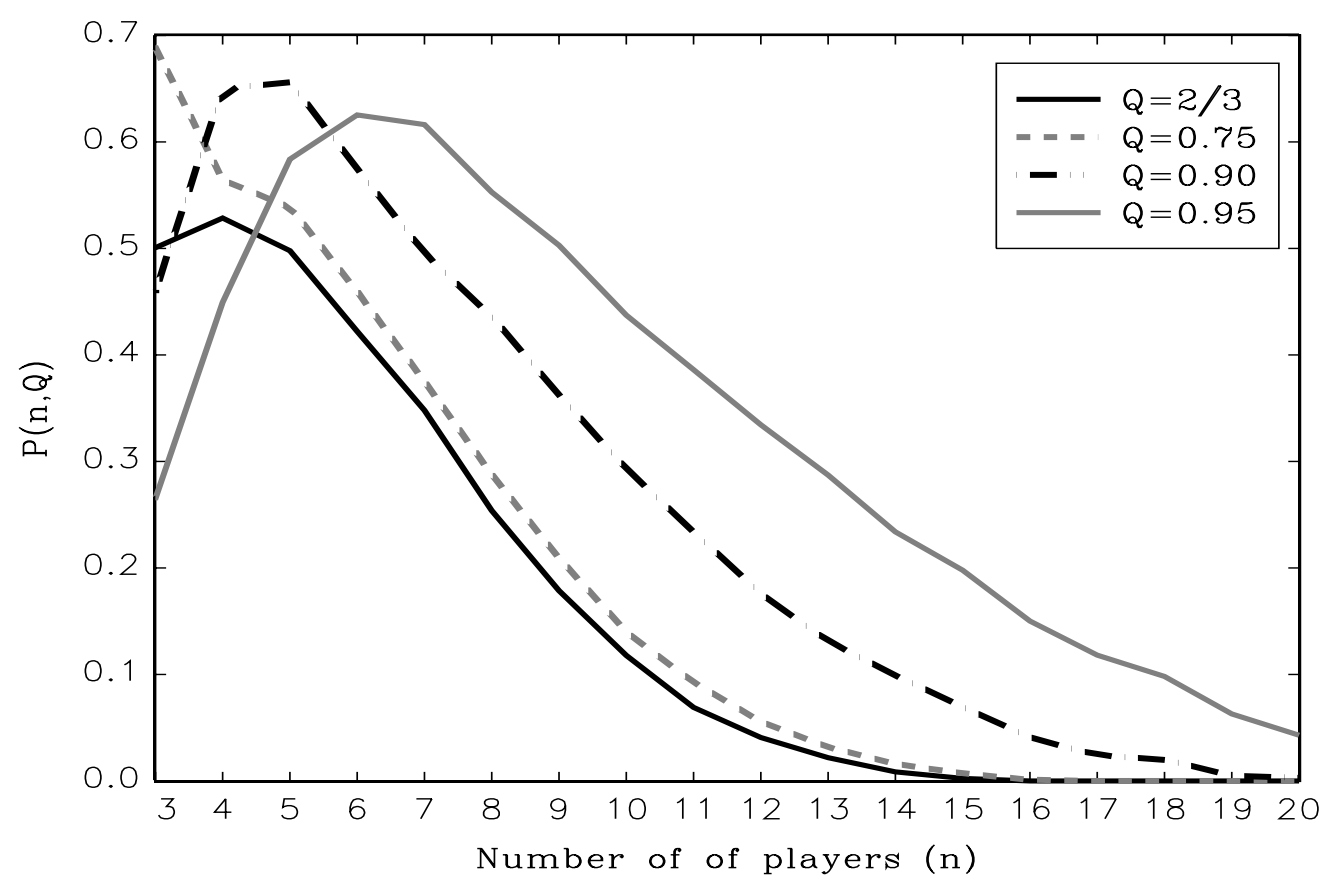

Figure 3 - Penrose's law when $w$ tends to infinity

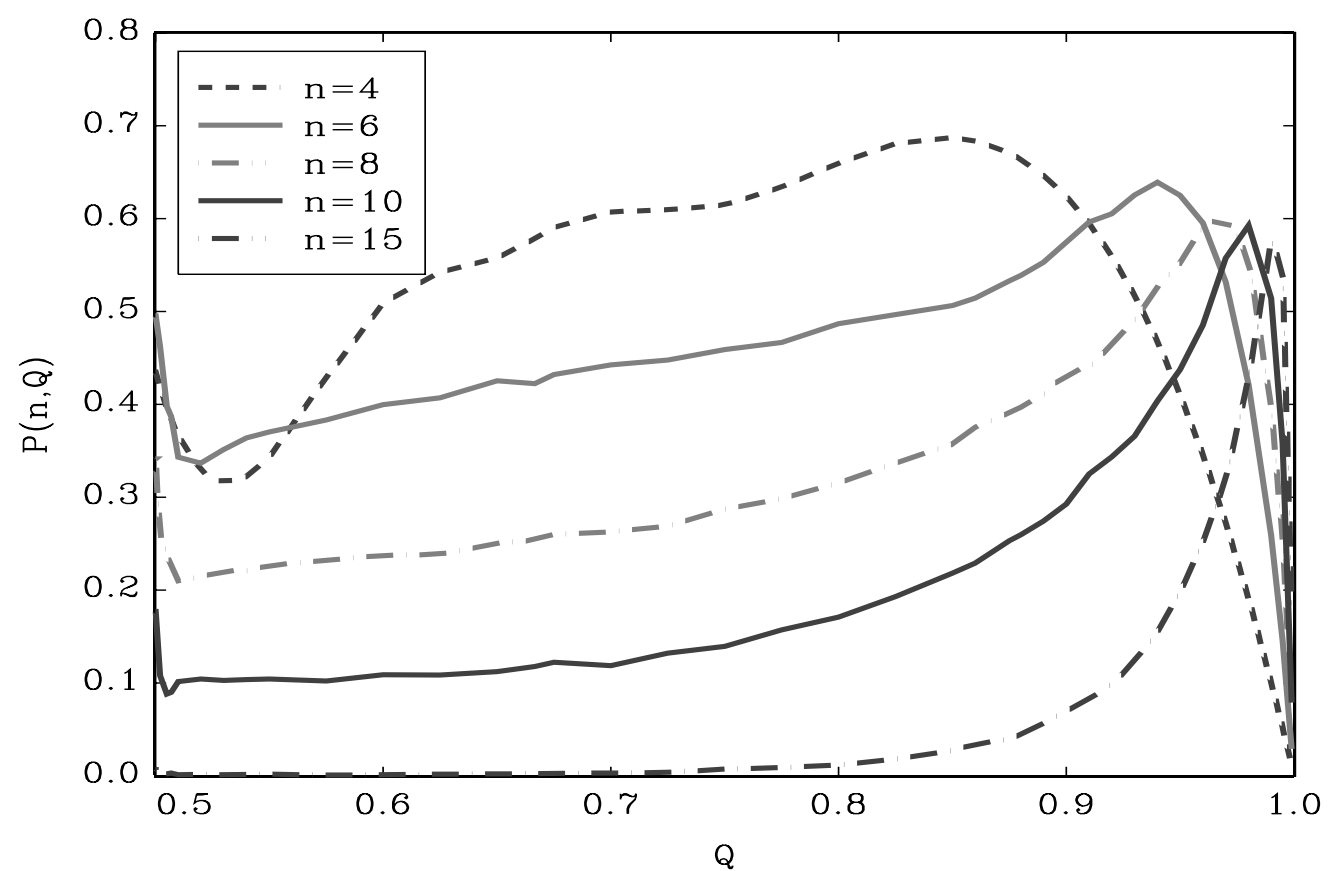

FiguRE 4 - Limiting probability of having at least one dummy player for 4 to 15 players 


\section{Conclusion and final remarks}

The main conclusions of our study can be summarized as follows.

1) Barthélémy et al. (2019) have shown that the probability of having a dummy player can be surprisingly high and is very sensitive to the choice of the quota in voting games with a small number of players. Our results confirm this conclusion for voting games with a larger number of players.

2) As suggested in Barthélémy et al. (2019), we conclude that the choice of a quota close to 1 (e.g. 0.95), that could be suggested by the observation that there is no dummy player for $Q=1$, would be a serious mistake: for $Q$ close to 1 , the risk of a dummy player would be more likely maximized rather than minimized.

2) In order to minimize the probability of having dummy players, it is advisable to choose a quota between 0.50 and 0.55 .

4) The probability of having a dummy first increases then decreases with the number of players, whatever the quota value, suggesting that the Penrose's law holds for $Q>1 / 2$. It is worth noticing, however, that this convergence towards 0 is much slower with high values of $Q$ than with small values. To illustrate, with 15 players, the probability of a dummy player is less than $0.25 \%$ for $0.5<Q<0.66$ and is still about $43 \%$ for $Q=0.98$.

5) Our conclusions have been obtained by assuming that the weighted voting games are generated via the IAC probabilistic model. Of course, this model does not constitute the only possibility for generating random games. It is therefore logical to ask whether the main phenomena we have observed arise with other distributions of the weighted voting games. To obtain some insights on this issue, we have run some further simulations based on an alternative probabilistic model. With this model, that we call NORM, the weight vectors $\left(w_{1}, w_{2}, \ldots, w_{n}\right)$ are generated by picking $n$ numbers in a uniform distribution on $[0,1]$ and then normalizing these numbers. We then proceed exactly as we did with the IAC model. The results we obtain via this method for the "infinite" case $(w=9999)^{8}$ are displayed in Table 5. We obtain the same qualitative conclusions as before, which suggests that our conclusions could be relatively robust.

8. The results we have obtained for smaller values of $w$ are available from the authors upon request. 
Table 5. Simulated Limiting Probability of having at least one dummy for 3 to 15 players

$$
w=9 \text { 999, } 10000 \text { simulations (in\%) (NORM) }
$$

\begin{tabular}{|c|ccccccccccccc|}
\hline & \multicolumn{10}{|c}{ Number of players } \\
\hline Quota $Q$ & 3 & 4 & 5 & 6 & 7 & 8 & 9 & 10 & 11 & 12 & 13 & 14 & 15 \\
\hline 0.50 & 0 & 61.68 & 43.47 & 39.00 & 28.05 & 20.70 & 13.26 & 8.86 & 4.92 & 2.26 & 1.39 & 0.46 & 0.18 \\
0.51 & 0.28 & 51.98 & 33.15 & 26.00 & 16.11 & 11.27 & 7.37 & 4.51 & 2.56 & 0.92 & 0.53 & 0.14 & 0.03 \\
0.52 & 1.03 & 44.54 & 28.22 & 23.45 & 16.82 & 11.14 & 7.05 & 4.67 & 2.57 & 1.09 & 0.50 & 0.12 & 0.03 \\
0.55 & 5.96 & 33.04 & 28.36 & 23.12 & 17.10 & 11.09 & 7.22 & 4.45 & 2.47 & 1.11 & 0.54 & 0.23 & 0.03 \\
0.60 & 21.61 & 34.32 & 35.71 & 25.35 & 18.48 & 12.56 & 7.84 & 4.67 & 2.82 & 1.23 & 0.72 & 0.19 & 0.02 \\
0.65 & 47.16 & 34.79 & 35.23 & 26.17 & 19.22 & 13.32 & 8.92 & 5.64 & 3.30 & 1.68 & 0.82 & 0.37 & 0.11 \\
$2 / 3$ & 58.05 & 38.12 & 33.69 & 26.96 & 18.93 & 13.37 & 8.98 & 6.00 & 3.51 & 1.76 & 0.94 & 0.35 & 0.14 \\
0.70 & 71.97 & 40.80 & 32.92 & 31.20 & 21.30 & 15.07 & 10.24 & 6.90 & 4.12 & 2.18 & 1.15 & 0.69 & 0.11 \\
0.75 & 70.93 & 47.69 & 37.28 & 29.62 & 25.32 & 18.37 & 12.87 & 8.97 & 5.559 & 3.31 & 1.90 & 0.81 & 0.50 \\
0.80 & 60.45 & 65.55 & 42.43 & 35.11 & 27.94 & 21.81 & 16.66 & 12.95 & 8.259 & 5.32 & 3.56 & 1.97 & 1.16 \\
0.85 & 46.35 & 65.94 & 59.22 & 43.09 & 35.37 & 29.08 & 22.32 & 17.66 & 13.94 & 9.38 & 6.67 & 4.48 & 2.77 \\
0.90 & 30.19 & 51.98 & 63.09 & 60.80 & 50.87 & 40.97 & 34.20 & 28.63 & 23.34 & 18.05 & 14.07 & 10.66 & 7.73 \\
0.95 & 15.00 & 28.34 & 41.28 & 53.08 & 59.73 & 60.33 & 57.41 & 52.12 & 45.30 & 39.25 & 34.72 & 30.36 & 25.50 \\
0.98 & 5.91 & 12.12 & 18.84 & 26.44 & 34.05 & 41.92 & 48.39 & 53.41 & 56.83 & 57.32 & 58.35 & 56.32 & 52.82 \\
0.99 & 2.95 & 5.95 & 9.49 & 13.90 & 18.60 & 23.73 & 29.39 & 35.07 & 40.21 & 43.75 & 48.29 & 50.75 & 54.27 \\
\hline
\end{tabular}

\section{References}

Barthélémy F., Lepelley D. and Martin M. (2013), On the likelihood of dummy players in weighted majority games, Social Choice and Welfare 41, 263-279.

Barthélémy F., Lepelley D. and Martin M. (2019), Dummy players and the quota in weighted voting games, Working Paper, THEMA

Barthélémy F., Martin M. and Tchantcho B. (2011), Some conjectures on the two main power indices, Working paper 2011-14, THEMA.

Chang P.-L., Chua V.C.H. and Machover M. (2006), L.S. Penrose's limit theorem: Tests by simulation, Mathematical Social Sciences 51, 90-106.

Courtin S., Mbih B. and Moyouwou I. (2014), Are Condorcet procedures so bad according to the reinforcement axiom?, Social Choice and Welfare 42, 927-940.

Diss M., Louichi A., Merlin V. and Smaoui H. (2012), An example of probability computations under the IAC assumption : The stability of scoring rules, Mathematical Social Sciences 64, 57-66.

Felsenthal D. and Machover M. (1998), The Measurement of Voting Power. Theory and Practice, Problems and Paradoxes, Publishing, UK.

Gerhlein W. and Lepelley D. (2017), Elections, Voting Rules and Paradoxical Outcomes, Springer. 
Leech D. (2002), Power indices as an aid to institutional design: the generalized apportionment problem, Warwick Economic Research Papers, $n^{\circ} 648$.

Lepelley D., Louichi A. and Smaoui H. (2008), On Ehrhart polynomials and probability calculations in voting theory, Social Choice and Welfare 30, 363-383.

Lindner I. and Machover M.(2003), L.S. Penrose's limit theorem: proof of some special cases, Mathematical Social Sciences 47, 37-49.

Nijenhuis A. and Wilf H. (1978), Combinatorial Algorithms for Computers and Calculators, 2nd ed. New York: Academic Press,

(http ://www.math.upenn.edu/ wilf/website/CombinatorialAlgorithms.pdf).

Penrose L.S. (1946), The elementary statistics of majority voting, Journal of the Royal Statistical Society 109, 53-57.

Penrose L.S. (1952), On the objective study of crowd behavior, London : H.K. Lewis and Co. Straffin P.D. (1994), Power and stability in politics, Chapter 32, Aumann. R.J and Hart S., eds, Handbook of Game Theory, vol 2, North-Holland, Amsterdam, The Netherlands. 